\section{Der Einfluss von Desoxyribonuklease (DNase) auf die Grösse des penicillin-(Pc)resistenten Anteils einer Population von M. pyogenes var. aureas Sg 511}

Die Induktion einer Pc-Resistenz oder anderer genetischer Faktoren durch eine dem Suspensionsmedium von Keimen zugesetzte DNS wird bekanntlich durch DNase aufgehoben. Nicht beschrieben wurde aber der mögliche Einfluss von DNase als solcher auf den pc-resistenten Anteil einer Keimpopulation. Untersucht wurde deswegen der Einfluss von kristalliner DNase (Dr. ScruCHARDT, München und C. Roth, Karlsruhe) auf die Grösse des pc-resistenten Anteils von Staph. aureus Sg 511.

Der Teststamm hatte nach dem üblichen Dilutionstest eine mittlere Sensibilität von $0,04 \mathrm{E} \mathrm{Pc/ml}$. Zur Lösung von DNase bzw. zur Suspension der von einer $16 \mathrm{~h}$ alten Agarkultur stammenden und zweimal gewaschenen Testkeime diente eine $0,01 M$-Lösung von $\mathrm{MgCl}_{2} \cdot 7 \mathrm{H}_{2} \mathrm{O}$. Gleiche Volumina der Keimsuspension (mikroskopisch eingestellt auf $10^{10}$ Zellen je $\mathrm{ml}$ ) und der Lösung von kristalliner DNase $(400 \mu \mathrm{g} / \mathrm{ml})$ wurden gemischt und $20 \mathrm{~min}$ im Wasserbad bei $37^{\circ} \mathrm{C}$ erwärmt. Zur Bestimmung der "Lebendkeimzahl» (Koloniezahl) wurden davon dann 10 Petrischalen (Nähragar) mit je $0,3 \mathrm{ml}$ nach Vorverdünnung der Keimsuspension auf $0,2 \cdot 10^{-6}$ beschickt. Zur Bestimmung des resistenten Anteils (Koloniezahl) wurden weitere 10 Platten mit pc-haltigem Nähragar mit je $0,3 \mathrm{ml}$ dieser Keimsuspension ohne Vorverduinnung beimpft.

\begin{tabular}{|c|c|c|c|c|c|c|}
\hline \multirow{3}{*}{$\int_{0}^{\frac{1}{3}}$} & \multirow{3}{*}{ Ansatz } & \multicolumn{2}{|c|}{\begin{tabular}{|c|c|}
\multicolumn{2}{|c|}{ Koloniezahl je $\mathrm{ml}$} \\
Gesamt & $\mathrm{Re}$ \\
\end{tabular}} & \multirow{3}{*}{$\begin{array}{c}\text { Re/10 } \\
\text { Gesamt } \\
\pm \% \\
\end{array}$} & \multirow{2}{*}{\multicolumn{2}{|c|}{$\begin{array}{l}\text { Vergleich von } \mathrm{Re} / 10 \\
\text { Gesamt bei Kontrolle } \\
\text { und Versuch }\end{array}$}} \\
\hline & & $\times 10^{R}$ & -8 & & & \\
\hline & & $\pm \%$ & - & & $t$ & $P$ \\
\hline 1 & $\begin{array}{l}\text { DNase } \\
\text { aktiv } \\
\text { DNase } \\
\text { inaktiv }\end{array}$ & $\begin{array}{r}403,3 \\
+\quad 36,8 \\
458,3 \\
\pm \quad 38,2\end{array}$ & $\begin{array}{r}759,7 \\
+\quad 18,3 \\
1236,3 \\
+\quad 13,4\end{array}$ & $\begin{array}{r}1883,6 \\
+\quad 18,3 \\
2697,6 \\
+\quad 13,4\end{array}$ & 5,1492 & $<0,001$ \\
\hline 2 & $\begin{array}{l}\text { DNase } \\
\text { aktiv } \\
\text { DNase } \\
\text { inaktiv }\end{array}$ & $\begin{array}{r}421,7 \\
\pm \quad 20,9 \\
531,7 \\
\pm \quad 17,1\end{array}$ & $\begin{array}{r}740,3 \\
\pm \quad 14,7 \\
1212,3 \\
\pm \quad 20,2\end{array}$ & $\begin{array}{r}1757,0 \\
\pm \quad 14,7 \\
2280,2 \\
\pm \quad 20,2\end{array}$ & 3,1247 & $\begin{array}{c}<0,02 \\
(>0,01)\end{array}$ \\
\hline
\end{tabular}

Die Konzentration des verwandten Na-Salzes von PC-G wurde zur guten statistischen Erfassung so eingestellt, dass die Zahl der je Platte gewachsenen resistenten Kolonien mindestens 150 betrug, das heisst bei Versuch $1=$ $0,06 \mathrm{E} \mathrm{Pc} / \mathrm{ml}$ und bei Versuch $2=0,04 \mathrm{E} \mathrm{Pc} / \mathrm{ml}$. Als Kontrolle diente derselbe Ansatz mit der gleichen Keimsuspension, wobei lediglich die D-Nase-Lösung vorher $60 \mathrm{~min}$ auf Kochtemperatur gehalten worden war. Die
Auszählung der auf Agar ohne und mit Pc-Zusatz gewachsenen Kolonien erfolgte nach $24 \mathrm{bzw} .48 \mathrm{~h}$ Bebrütung bei $37^{\circ} \mathrm{C}$. Die Zahl der resistenten Kolonien einer jeden Platte (Re) wurde auf die mittlere Gesamtzahl der Kolonien bezogen und danach die Zahl der Re je $10^{9}$ insgesamt gewachsenen Kolonien errechnet. Mit Students $t$-Test wurden die so bei Versuch und Kontrolle ermittelten Werte unter Berücksichtigung der Streuung statistisch verglichen.

Aus der Aufstellung ist zu entnehmen, dass durch $\mathrm{Zu}$ gabe von kristalliner DNase der pc-resistente Anteil der Keimpopulation in statistisch signifikanter Weise kleiner wird, wenn man als Kriterium der Signifikanz eine Wahrscheinlichkeit von $2 \%$ für die Zufallsbedingtheit der Differenz annimmt.

\section{G. Gillissen}

Hygiene-Institut der Universität Mainz, 5. Januar 1959.

\section{Summary}

The number of penicillin-resistant organisms among a certain population of Staph, auveus can be reduced by treatment with crystalline desoxyribonuclease. These results are statistically significant.

\section{IN FORMATIONES}

\section{Congressus}

GERMANY

\section{Geochemical Symposium}

Göttingen, August $21^{\text {st }}$ to $24^{\text {th }}, 1959$

The Commission on Geochemistry of the International Union of Pure and Applied Chemistry will be holding a Symposium on:

(1) Stable Isotopes in Geochemistry;

(2) Long-living Natural Isotopes;

(3) Geochemistry of the Halogenes;

(4) Bio-Geochemistry

in Göttingen (Germany) on August 21st and 22nd, 1959, followed by Excursions on August $23^{\text {rd }}$ and $24^{\text {th }}, 1959$.

Details may be obtained from Professor Dr. Correns, Sedimentpetrographisches Institut, Göttingen, Lotzestrasse 13. 